\title{
Application of High Resolution lon Mobility Spectrometers for Fast Trace Gas Analysis
}

\author{
A. Kirk, M. Allers, P. Cochems, J. Langejuergen, S. Zimmermann \\ Institute of Electrical Engineering and Measurement Technology, Department of Sensors and \\ Measurement Technology, Leibniz University, Hannover, Germany \\ kirk@geml.uni-hannover.de
}

\begin{abstract}
:
Ion mobility spectrometers (IMS) are a widely used tool for fast trace gas detection, generally offering excellent limits of detection (LoD) in a short amount of time, but only mediocre resolution. In this work, we demonstrate an approach to vastly increase the resolution without sacrificing limits of detection or increasing the complexity of the measurement setup. Based on these findings, a compact ion mobility spectrometer with a drift length of less than $10 \mathrm{~cm}$ has been constructed. It offers a resolution of above 180 in both positive and negative mode while maintaining detection limits in the ppt $_{v}$ range for an averaging time of less than a second. This resolution is e.g. sufficient to separate the two isomers sec-butylamine and tert-butylamine, even though their collision cross sections and therefore drift times differ by less than one percent. Furthermore, analysis of the spectrum of chlorine shows a change in peak shape which could indicate the beginning separation of the two stable isotopes.
\end{abstract}

Key words: Ion mobility spectrometry, high resolution, compact, isomers, isotopes

\section{Introduction}

Ion mobility spectrometers (IMS) characterize substances by studying the motion of their ions through a neutral gas under the influence of an electric field. Originally deployed as detectors for chemical warfare agents, explosives and other hazardous substances, they have become a widespread chemical sensor in science and industry due to their unique advantages [1],[2]. Prominent examples for their new areas of use are quality control for food [3] and medication [4] or breath gas analysis [5]. Furthermore, IMS-MS-couplings are popular in bioanalysis due to their ability to resolve isomers which is not directly possible by standalone MS [6]. Ion mobility spectrometers offer fast and sensitive detection of a wide range of substances with a single instrument while using a relatively simple and therefore cost-efficient instrumentation. One of their main disadvantages however is a rather limited resolution, reducing their ability to deal with complex samples containing many substances. Typical resolution values, which are defined as the ratio of the drift time and the full width at half maximum of a peak, range from 20 to 60 for commercially available devices [7].

To understand these traits better, taking a look at the origins of the namesake ion mobility $\mathrm{K}$ as shown by equation (1) is helpful. It is along with several environmental factors a function of the reduced mass calculated from the ion mass $m$ and the neutral mass $M$ as well as their momentum transfer collision cross section $\Omega$.

$$
K \propto \sqrt{\frac{1}{m}+\frac{1}{M}} \frac{1}{\Omega}
$$

This is both an important strength and weakness of ion mobility spectrometers. As they separate in both mass and size, isomers can be differentiated and ultra-high resolution devices are theoretically able to separate even isotopes. However, the relationships in equation (1) also mean that the ion mobility of different substances differs much less than for example their mass. Thus, the separation space of IMS is relatively small. Combined with the mentioned low resolution, this leads to difficulties when trying to separate samples with various substances in them, as they can be expected from many real-world applications. The improvement of the system resolution in order to mitigate these problems has therefore been an ongoing goal of the IMS community.

\section{Towards Ultra-High Resolution}

The first step towards being able to build higher resolution ion mobility spectrometers is gaining an understanding of the different processes occurring during a measurement and how to optimize them. 


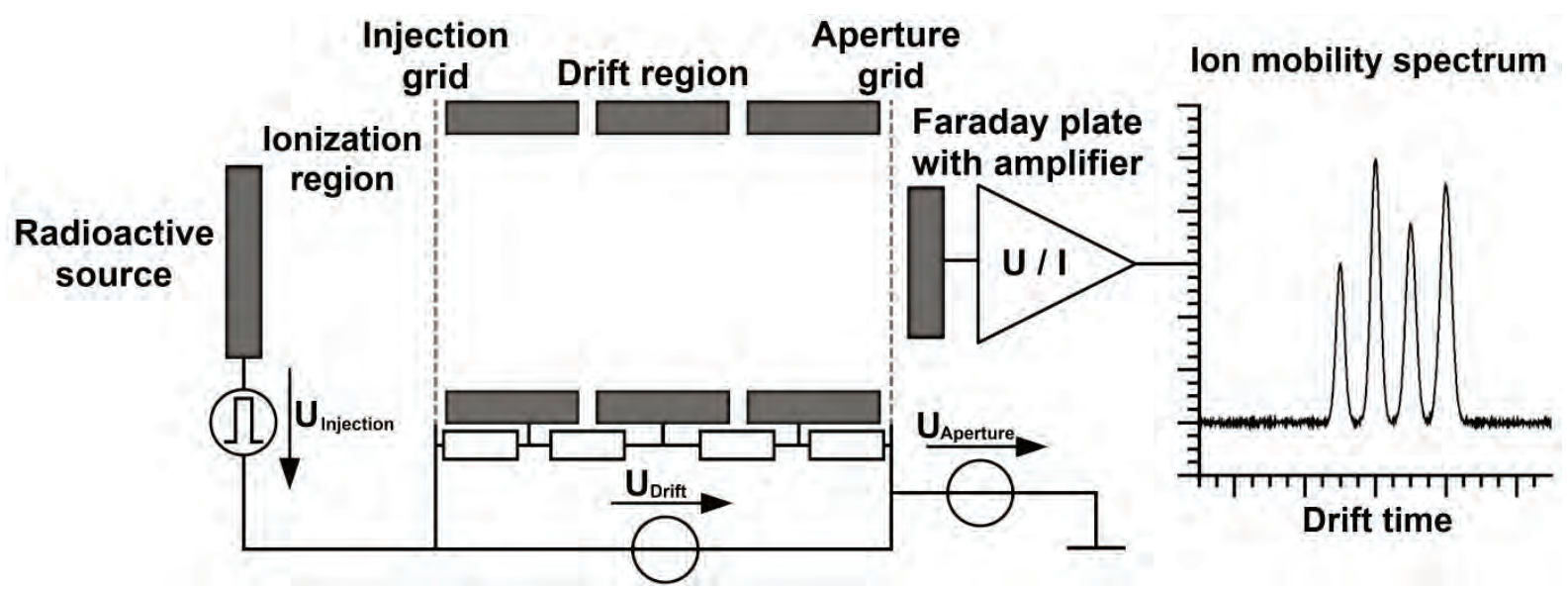

Fig. 1. Schematic diagramm of a drift tube ion mobility spectrometer

Fig. 1 shows the typical setup of a drift tube IMS, consisting of three distinct regions. From left to right, they are the ionization region, the drift region and the faraday plate which serves as the detector. In the ionization region reactant ions, which are mostly hydronium water clusters and oxygen water clusters, are generated by a reaction chain initialized by electron impact [1]. In most IMS, radioactive sources such as ${ }^{3} \mathrm{H}$ or ${ }^{63} \mathrm{Ni}$ are used to provide primary electrons. The analyte gas which is to be characterized is also directed into the ionization region, where further chemical reactions between reactant ions and analyte molecules form different analyte ions. The second region is the drift region, which is separated from the ionization region by a metallic grid. Upon a certain point in time, ions from the ionization region are injected into the drift region. In our setup, this is accomplished by raising the electric potential of the radioactive source for a short period of time, but different ion shutter principles exist. The injection process both determines the amount of charge entering the drift region and the timeframe across which it is distributed. Typically, trying to achieve a shorter pulse will result in less charge and vice-versa. The ions then travel through the drift region under the influence of the electric drift field created by the metallic drift rings. Each ion species moves with a speed proportional to their mobility, resulting in a separation of different species. During their journey, the ion packets broaden due to several effects, among them mainly diffusion, but also coulomb repulsion and inhomogeneities of the drift field. At the end of the drift region, the ions are discharged on a faraday plate and the resulting current is amplified by a transimpedance amplifier. Plotting this current over the time elapsed since the ion injection results in the characteristic IMS spectrum. The faraday plate is shielded from the electric field of the ion clouds by the so-called aperture grid to prevent peak broadening. The bandwidth of the amplifier determines both the resulting distortion of the peak as well as the noise present in the spectrum. Similar to the compromise during the injection process, less distortion comes at the price of increased noise.

This is the measurement process which needs to be optimized in order to achieve higher resolution. However, optimizing for resolution only is not sufficient, as a measurement instrument which could theoretically differentiate many substances simultaneously, but is unable to detect them, is of little use. Therefore, the limits of detection (LoD) need to be optimized at the same time. However, for the optimization it is more convenient to use the signal-to-noise-ratio (SNR) achieved with a given amount of ions in a certain timeframe, as the ionization process is not changed and can therefore be left out. A higher SNR leads to proportionally lower LoDs.

Fig. 2 illustrates the problems connected with this task by showing three simulated spectra. The first spectrum is an example of a typical IMS spectrum such as it is achieved by most instruments. It has a good SNR due to the sensitivity of the chemical ionization, but the resolution is rather limited and the several peaks present cannot be separated. The second spectrum is the result of the typical experimental optimization procedure for resolution enhancement, which is simply reducing the width of the initial ion packet injected. However, as mentioned before, this also reduces the amount of charge injected into the drift region and therefore the signal intensity. Due to this problem, reaching a high resolution this way is not realistic. Thus, a more sophisticated optimization process is needed, which will be explained later in this work. The final result of this approach is already shown in the third spectrum, providing high resolution sufficient to separate the different peaks while maintaining the high signal-to-noise-ratio. 

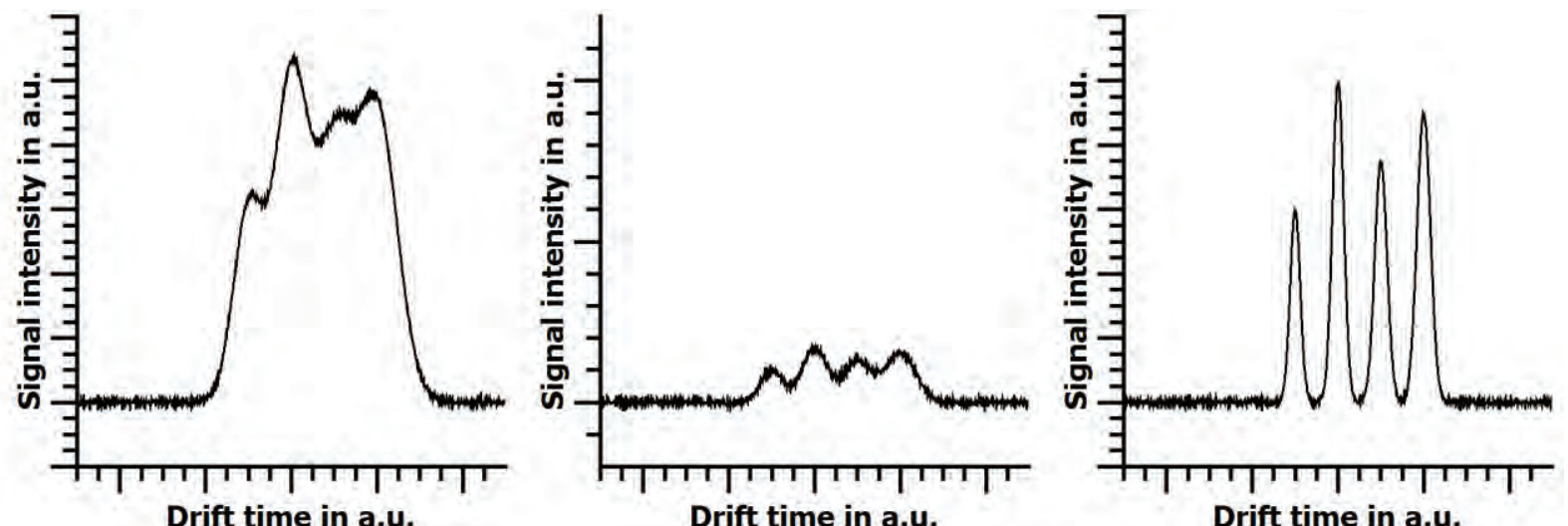

Fig. 2. Simulated peaks during the optimization process. From left to right: Original spectrum, spectrum achieved by simple reduction of initial width, spectrum achieved by the optimization process from [8]

For deriving this optimization method, we used both analytical and numerical methods in order to gain a thorough understanding of the processes inside a drift tube. Analytical models are best used to gain an understanding of the relationships between different parameters of the device, while numerical simulation are useful to minimize non-idealities which are not visible from measurements or simple models. In [8], we presented the analytical model we built for system optimization. It includes equations for both resolution and SNR and describes the peak broadening and ion loss during the injection, the diffusive broadening during the flight and the noise and peak broadening by the amplification. In the end, it provided us with two equations which describe how an IMS needs to be built for maximum performance. In these equations, $L_{D}$ describes the length of the drift region, $w_{I n j}$ the width of the ion packet resulting from the injection process and $w_{\text {Amp }}$ the width of the ion packet resulting from the amplification distortion.

$$
\begin{aligned}
& R \propto \sqrt[3]{\frac{L_{D}{ }^{2}}{\sqrt{w_{I n j}{ }^{2}+w_{A m p}{ }^{2}}}} \\
& S N R \propto \frac{w_{I n j} w_{A m p}}{w_{I n j}{ }^{2}+w_{A m p}{ }^{2}}
\end{aligned}
$$

According to equation (2), the system resolution depends on the length of the drift tube and the geometric sum of the half-widths created by injection and amplification. As shown by equation (3), there is no remaining influence of the drift length on the SNR, as all effects related to it cancel each other out. It depends only on the matching between $\mathrm{w}_{\mathrm{Inj}}$ and $\mathrm{w}_{\mathrm{Amp}}$, as it can be seen that equation (3) becomes a constant if they are chosen to be equal. Therefore, two possible ways for the construction of a high resolution device exist. However, as vastly increasing the drift length leads to both costly and unwieldy instruments, we have chosen to build an optimized IMS system with fast and matched injection and amplification to achieve high performance. The disadvantage of this approach is, as shown in [8], that the system becomes more susceptible to non-idealities such as field inhomogeneities inside the drift tube. Thus, we used an extensive finite element model built in COMSOL [9] in order to analyze and eliminate non-idealities in our drift tube. Fig. 3 shows one result of one of these simulations. In the left-hand half, the electric field lines inside the drift tube are shown. It is obvious, that the homogeneity of the field decreases with increasing vicinity to the drift rings, which are marked by the black lines on the left and right edge of the picture. The effect of these inhomogeneities becomes visible in the right-hand side of the figure, which displays the ion distribution inside the drift tube. While the ions close to the center will arrive at the detector at the same time, the ions which move through the field inhomogeneities at the edges are lagging behind and will therefore reach the detector at a later point in time. This causes peak broadening, which leads to loss of resolution and must therefore be avoided. With the aid of the simulation it is possible to design a drift tube where these and other effects do not possess a significant influence on the system performance.

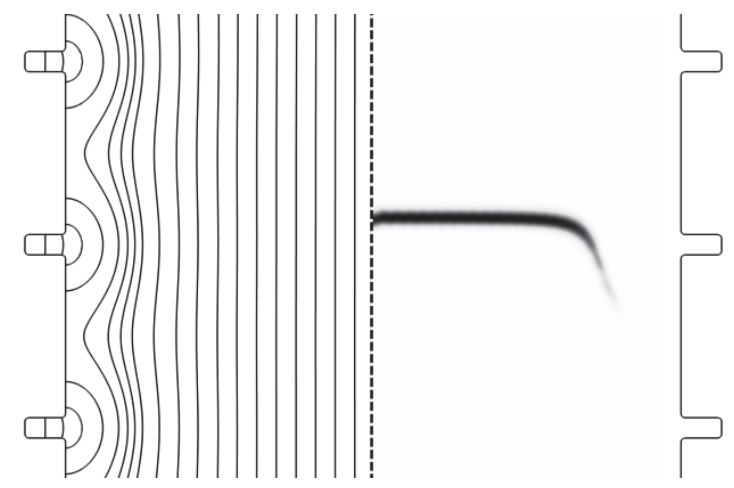

Fig. 3. Simulation of a drift tube IMS. The left half shows the electric field lines, while the right half shows the ion distribution during the flight. 


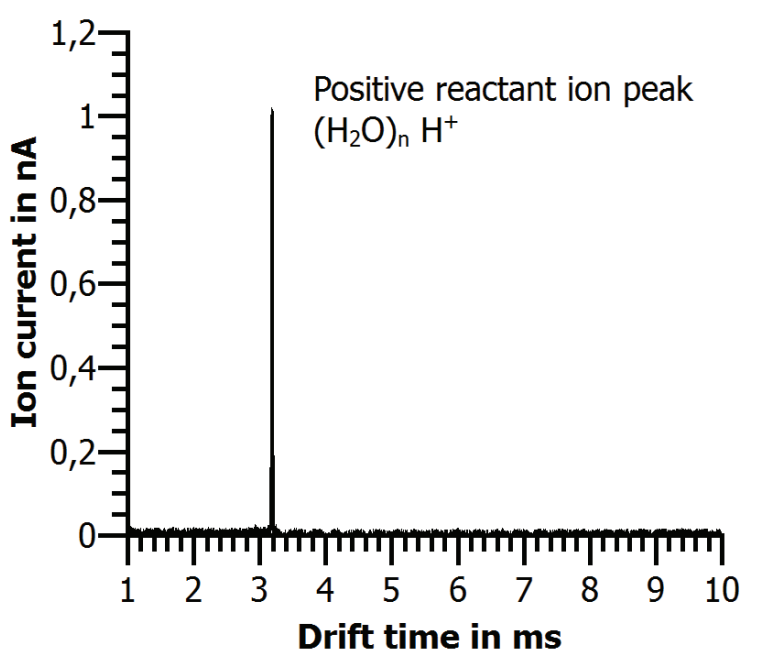

Fig. 4. Positive reactant ion peak $\left(R I P^{+}\right)$with a resolution of $183 \pm 0,5$.

\section{Experimental Setup}

In order to obtain a system with the needed specifications, we designed and built most components of the measurement setup at our institute. The drift tube layout follows the basic design shown in Fig. 1, with ionization region, injection grid, drift region, aperture grid and faraday plate. The drift rings and all other conducting parts are made from stainless steel, while PEEK is used for insulators and PTFE for seals. A radioactive ${ }^{3} \mathrm{H}$-source provides reactant ion generation. Purified air is used as both drift gas and sample carrier. All operating parameters are summarized in Tab.1.

Tab. 1: IMS operating parameters

\begin{tabular}{|c|c|}
\hline Parameter & Value \\
\hline Drift length & $98 \mathrm{~mm}$ \\
\hline Drift region diameter & $15 \mathrm{~mm}$ \\
\hline Source diameter & $10 \mathrm{~mm}$ \\
\hline Source activity & $300 \mathrm{MBq}$ \\
\hline Injection voltage & $1900 \mathrm{~V}$ \\
\hline Injection time & $5 \ldots 350 \mathrm{\mu s}$ \\
\hline Repetition rate & $44 \mathrm{~Hz}$ \\
\hline Drift voltage & $13 \mathrm{kV}$ \\
\hline Aperture voltage & $100 \mathrm{~V}$ \\
\hline Drift gas flow & $250 \mathrm{mls} / \mathrm{min}$ \\
\hline Sample gas flow & $5 \mathrm{mls} / \mathrm{min}$ \\
\hline Dew point & $-82^{\circ} \mathrm{C}$ \\
\hline drift gas and sample carrier & $(0,4 \mathrm{ppm} \mathrm{v}$ water \\
\hline vapour concentration $)$
\end{tabular}

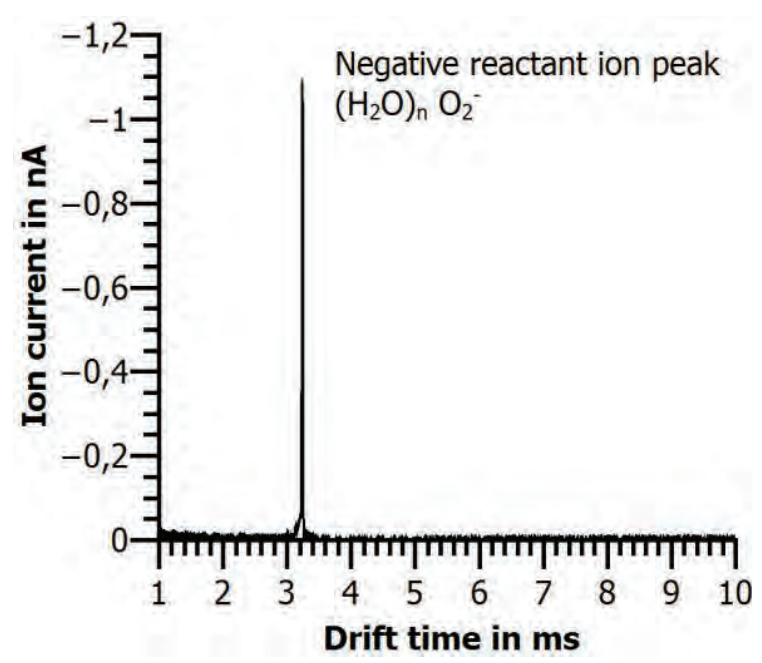

Fig. 5. Negative reactant ion peak $\left(R I P^{-}\right)$with a resolution of $183 \pm 0,5$.

As the presented design approach demands that the speeds of ion injection and signal amplification are matched, both the high voltage pulser used for ion injection and the transimpedance amplifier connected to the faraday plate are critical parts of the measurement setup. The high voltage pulser is able to switch voltages of $2,2 \mathrm{kV}$ within $100 \mathrm{~ns}$ while isolating a potential difference of $25 \mathrm{kV}$ against ground. The fast transimpedance amplifier possesses a rise time of $5,8 \mu \mathrm{s}$, which corresponds well to the minimum ion packet width achievable with our high voltage pulser. All other control electronics were also developed at our institute, but their characteristics are not of direct importance to the measurement setup. The only components which were not custom-built are the high voltage power supplies (FUG HCP 35-20000) and the oscilloscope (Agilent MSO9404A) used to capture the spectra.

All chemicals used in the experiments were purchased from Sigma-Aldrich. In order to generate the desired analyte concentration, a permeation tube containing the substance was placed in a permeation oven set to $35^{\circ} \mathrm{C}$ with a constant flow of $600 \mathrm{mls} / \mathrm{min}$ purified air. A certain fraction of this gas was then mixed with purified air to achieve the desired concentration.

\section{Results and Discussion}

Fig. 4 and Fig. 5 show the reactant ion peaks measured in both positive and negative mode. In both cases, a resolution of 183 could be obtained. The $95 \%$ confidence interval of the $t-$ distribution calculated from repeat measurements is $\pm 0,5$, indicating that this resolution can be reached reliably. At the same time, the signal-to-noise-ratio for an averaging time of $100 \mathrm{~ms}$ is 126 for the $\mathrm{RIP}^{+}$and 135 for 


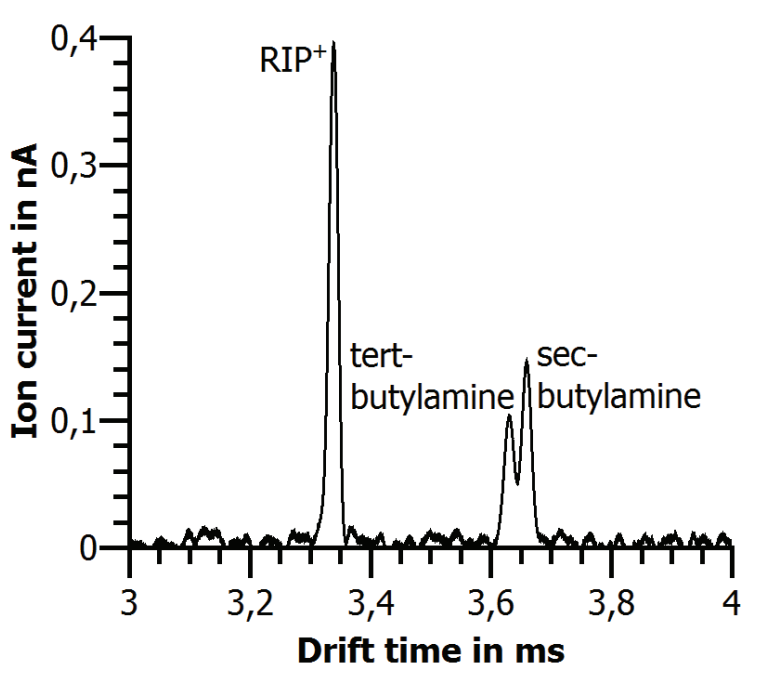

Fig. 6. Separation of tert- and sec-butylamine

the RIP'. As shown in [8], this results in a limit of detection for acetone of $580 \mathrm{ppt}_{\mathrm{v}}$ within the same timeframe. While the resolution of the reactant ion peaks is a good indicator for system performance, measuring samples containing substances which are difficult to separate is a good way to ensure that the high resolution actually results in better peak separation. One example for such a mixture is shown in Fig. 6. Sec-butylamine and tertbutylamine are two isomers of butylamine which exhibit drift times that differ by less than one percent, as they have the same mass and very similar collision cross sections. Thus, their separation is only possible at high resolution, confirming that the measured resolution of the reactant ion peak indeed translates to high separation power. Another example for two different ion species which are highly complicated to separate are isotopes. Due to the relationship between ion mass and ion mobility given by equation (1), the mass difference results in a much smaller ion mobility difference. For example, even though the mass difference between the two chlorine isotopes ${ }^{35} \mathrm{Cl}$ and ${ }^{37} \mathrm{Cl}$ is $5,71 \%$, the expected difference in ion mobility when using air as the drift gas is only $1,24 \%$. Furthermore, ions at atmospheric pressure typically do not exist as single ions, but clustered with water molecules [1]. The additional mass of these water molecules reduces the ion mobility difference between isotopes further, making their separation even more complicated. However, at the resolution of 183, the chlorine peak starts showing a significant tailing, which is not observable on other peaks. Fig. 7 shows the measured peak and a fit of two gaussian peaks, whose height ratio is the isotope ratio of chlorine. The agreement between their sum and the observed

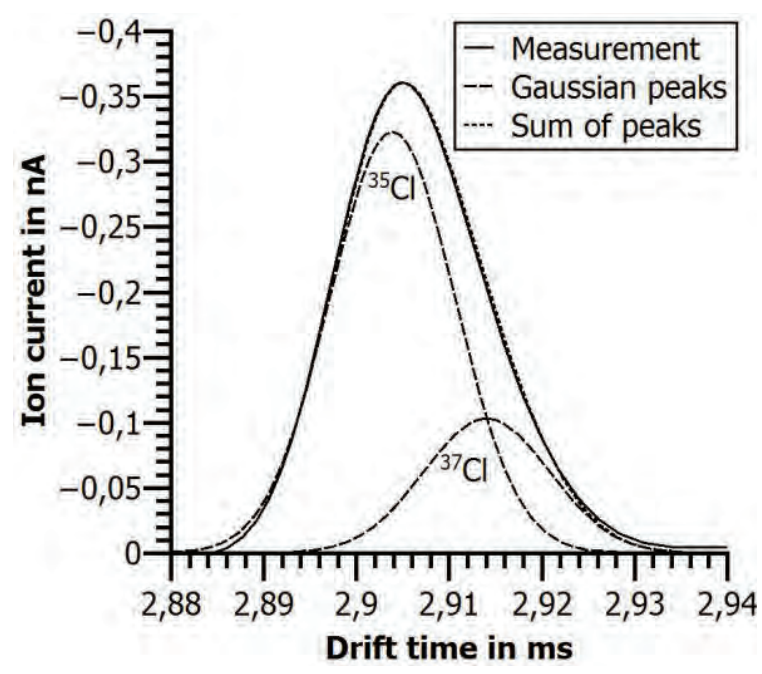

Fig. 7. Chlorine peak. Two gaussian peaks with height ratio equal to the isotope ratio were fitted to the measurement.

peak is excellent, indicating that the unique tailing of the chlorine peak may very well be the beginning of isotope separation in ion mobility spectrometers.

\section{Conclusions}

In this work, we demonstrated that through the combination of analytical and numerical optimization techniques, the construction of compact drift tube ion mobility spectrometers with both high resolution and low limits of detection becomes possible. Our current prototype achieves a resolution of 183 combined with detection limits in the ppt $\mathrm{v}_{\mathrm{v}}$-range for an averaging time of $100 \mathrm{~ms}$. As shown by the separation of sec- and tert-butylamine as well as the possible beginning separation of chlorine isotopes, this high resolution translates well to increased separation power. With this improvement in performance, ion mobility spectrometers take another step towards becoming a universal sensor for trace gas analysis tasks.

\section{References}

[1] Eiceman, G.A., Karpas, Z.; "Ion Mobility Spectrometry, 2nd Edition“; CRC Press, 2004

[2] Borsdorf H., Eiceman G.A.; "Ion Mobility Spectrometry: Principles and Applications"; Appl. Spectrosc. Rev., 2006, 41, 323-375

[3] Dwivedi, P., Matz, L. M., Atkinson, D. A., Hill, H. H. Jr; " Electrospray ionization-ion mobility spectrometry: a rapid analytical method for aqueous nitrate and nitrite analysis "; Analyst, 2004, 129, 139-144

[4] Budimir, N. , Weston, D. J., Creaser, C. S.; "Analysis of pharmaceutical formulations using atmospheric pressure ion mobility spectrometry combined with liquid chromatography and nano- 
electrospray ionization"; Analyst, 2007, 132,3440

[5] Kreuder, A.-E, Buchinger, H., Kreuer, S., Volk, Th., Maddula, S., Baumbach, J. I.;

"Characterization of propofol in human breath of patients undergoing anesthesia"; Int J for lon Mobility Spectrometry, 2011, 14, 167-175

[6] Pritchard, C., O'Connor, G., Ashcroft, A.E.; "The Role of Ion Mobility Spectrometry - Mass Spectrometry in the Analysis of Protein Reference Standards"; Anal Chem, 2013, 85, 7205-7212

[7] Cottingham, K.; "Ion Mobility Spectrometry Rediscovered", Anal Chem, 2003, 75(19), 435A439A

[8] Kirk, A., Allers, M., Cochems, P., Langejuergen, J., Zimmermann, S. „A Compact High Resolution Ion Mobility Spectrometer for Fast Trace Gas Analysis“; Analyst, 2013,138, 5200-5207

[9] Langejuergen, J., Cochems, P., Zimmermann, S.; „Results of a transient simulation of a drift tube ion mobility spectrometer considering charge repulsion, ion loss at metallic surfaces and ion generation"; Int J for Ion Mobility Spectrometry, 2012, 15(4), 247-255 